\title{
Vector integration and the Grothendieck inequality
}

\author{
by
}

AdAm Bowers (Columbia, MO)

\begin{abstract}
We relate the Grothendieck inequality to the theory of vector measures and show that the integral of an inner product with respect to a bimeasure can be computed in an iterative way. We then show an application to the theory of bounded linear operators.
\end{abstract}

1. Introduction. In 1956 Alexandre Grothendieck published his celebrated théorème fondamental de la théorie métrique des produits tensoriels [9], which Lindenstrauss and Pełczyński reformulated as a matrix inequality:

TheOREM 1.1 (Theorem 2.1 in [10, p. 279]). Let $\left(a_{i j}\right)_{i, j=1}^{\infty}$ be a scalar array such that

$$
\left|\sum_{i, j=1}^{n} a_{i j} t_{i} s_{j}\right| \leq M
$$

for all $n \in \mathbb{N}$, whenever $\left|t_{i}\right| \leq 1,\left|s_{j}\right| \leq 1$. Then for every set of vectors $\left(x_{i}\right)$ and $\left(y_{j}\right)$ in a Hilbert space,

$$
\left|\sum_{i, j=1}^{n} a_{i j}\left\langle x_{i}, y_{j}\right\rangle\right| \leq K_{G} M\left(\sup _{i \in \mathbb{N}}\left\|x_{i}\right\|_{H}\right)\left(\sup _{j \in \mathbb{N}}\left\|y_{j}\right\|_{H}\right)
$$

for all $n \in \mathbb{N}$, where $K_{G}$ is a universal constant and $\langle\cdot, \cdot\rangle$ denotes the inner product in the Hilbert space.

In an earlier work [3], the author derived a measure-theoretic analog of this theorem:

Theorem 1.2. Let $(X, \mathcal{A})$ and $(Y, \mathcal{B})$ be two measurable spaces and $H$ a separable Hilbert space with inner product $\langle\cdot, \cdot\rangle$. If $f$ and $g$ are bounded measurable functions taking values in $H$, then the scalar-valued function $\langle f, g\rangle: X \times Y \rightarrow \mathbb{R}$

$$
\langle f, g\rangle(x, y)=\langle f(x), g(y)\rangle, \quad(x, y) \in X \times Y,
$$

2010 Mathematics Subject Classification: 46G10, 28A10.

Key words and phrases: vector measures, bimeasures, Grothendieck inequality. 
can be integrated with respect to any $\mu \in F_{2}(\mathcal{A}, \mathcal{B})$, and

$$
\left|\int\langle f, g\rangle d \mu\right| \leq K_{G}\|f\|_{\infty}\|g\|_{\infty}\|\mu\|_{F_{2}},
$$

where $K_{G}$ is the Grothendieck constant.

We recall that $F_{2}(\mathcal{A}, \mathcal{B})$ is the collection of bimeasures on $\mathcal{A} \times \mathcal{B}$, that is, the space of set functions $\mu: \mathcal{A} \times \mathcal{B} \rightarrow \mathbb{R}$ which are countably additive in each argument separately. The norm on $F_{2}$ is given by

$$
\|\mu\|_{F_{2}}=\sup \left\{\left|\sum_{A \in \mathcal{E}, B \in \mathcal{F}} \mu(A, B) \epsilon_{A} \delta_{B}\right|: \mathcal{E} \in \pi_{X}, \mathcal{F} \in \pi_{Y}, \epsilon_{A}= \pm 1, \delta_{B}= \pm 1\right\},
$$

where $\pi_{E}$ denotes the collection of all finite measurable partitions of the set $E$.

When $X$ and $Y$ are discrete, the bimeasures correspond to the scalar arrays in Theorem 1.1. and $\|a\|_{F_{2}}$ represents the smallest $M$ satisfying (1.1). In this case, we denote the set of bimeasures on $X \times Y$ by $F_{2}(X, Y)$.

The integral in (1.3) was given by the following:

$$
\int\langle f, g\rangle d \mu=\lim _{N \rightarrow \infty} \int\left(\sum_{j=1}^{N} f_{j} \otimes g_{j}\right) d \mu,
$$

where $\left(f_{j}\right)_{j=1}^{\infty}$ and $\left(g_{j}\right)_{j=1}^{\infty}$ are the coordinate functions with respect to the orthonormal basis $\left(e_{j}\right)_{j=1}^{\infty}$ of $H$.

In this paper, we show that the integral in (1.4) can also be computed in the following way:

TheOREM 1.3. Let $(X, \mathcal{A})$ and $(Y, \mathcal{B})$ be measurable spaces and $H$ a separable Hilbert space with inner product $\langle\cdot, \cdot \cdot\rangle$. If $f$ and $g$ are bounded measurable functions taking values in $H$, then the integral $\int\langle f, g\rangle d \mu$ can be realized as an integral of an $H$-valued function with respect to an $H$-valued measure:

$$
\begin{aligned}
\int_{X \times Y}\langle f(x), g(y)\rangle \mu(d x, d y) & =\int_{X}\left\langle f(x), \nu_{g}(d x)\right\rangle \\
& =\int_{Y}\left\langle g(y), \nu^{f}(d y)\right\rangle,
\end{aligned}
$$

where

$$
\nu_{g}=\int_{Y} g(y) \mu(\cdot, d y) \quad \text { and } \quad \nu^{f}=\int_{X} f(x) \mu(d x, \cdot)
$$

are $H$-valued measures on $\mathcal{A}$ and $\mathcal{B}$, respectively, with the property that

$$
\left\|\nu_{g}\right\|_{M} \leq K_{G}\|\mu\|_{F_{2}}\|g\|_{\infty} \quad \text { and } \quad\left\|\nu^{f}\right\|_{M} \leq K_{G}\|\mu\|_{F_{2}}\|f\|_{\infty} .
$$

This theorem mirrors the situation in the scalar case: If $f \in L^{\infty}(X)$ and $g \in L^{\infty}(Y)$ (i.e., they are bounded scalar-valued measurable functions on $X$ 
and $Y$, respectively), then

$$
\int f \otimes g d \mu=\int_{X} f(x) \mu_{g}(d x)=\int_{Y} g(y) \mu^{f}(d y),
$$

where

$$
\mu_{g}=\int_{Y} g(y) \mu(\cdot, d y) \quad \text { and } \quad \mu^{f}=\int_{X} f(x) \mu(d x, \cdot)
$$

are measures of finite total variation. Furthermore,

$$
\left\|\mu_{g}\right\|_{M} \leq\|g\|_{\infty}\|\mu\|_{F_{2}} \text { and } \quad\left\|\mu^{f}\right\|_{M} \leq\|f\|_{\infty}\|\mu\|_{F_{2}},
$$

and so

$$
\left|\int f \otimes g d \mu\right| \leq\|f\|_{\infty}\|g\|_{\infty}\|\mu\|_{F_{2}} .
$$

(There exist unbounded functions $f$ and $g$ such that the iterated integrals on either side of (1.8) exist but are not equal; see [14, Example 5.3 on p. 123].) For a detailed treatment of the scalar case, see Chapter VI of [2].

The integrals in (1.5)-(1.7) involve Hilbert space-valued integrands and Hilbert space-valued measures. The integral of a Banach space-valued function with respect to a Banach space-valued measure is developed in Section2. This integral is valid for general Banach spaces, but Theorem 1.3 can hold in general only when the Banach space is a Hilbert space. (See Theorem 3.12.)

In Section 4, we demonstrate an application to bounded linear operators on $L^{p}$-spaces (Theorem 4.1).

Throughout this paper, $\mathbb{R}$ is the scalar field, but the results can be adapted to $\mathbb{C}$. Whenever $\langle\cdot, \cdot\rangle$ and $\|\cdot\|$ appear without subscript, they are understood to be the inner product and norm, respectively, on the Hilbert space $H$, unless specifically stated otherwise.

2. Vector measure theory. The study of Banach space-valued measures and integration has been undertaken by various authors (e.g., Bartle [1], Diestel and Uhl [4], Dinculeanu [5], Dobrakov [6], Pettis [12]). Generally, integration is considered by constructing $L^{1}$-type spaces for a given measure of finite variation or semivariation. Here, we consider integration with respect to any measure of finite variation. (Cf. the integral of Dunford [7].) For our integral, finite semivariation of the measure will suffice, but in the cases we consider, the two notions coincide (e.g., Proposition I.4.4 in [5]).

In this section, $X, Y$, and $Z$ denote Banach spaces, and $(K, \mathcal{F})$ is a measurable space. All Banach spaces are assumed to be separable. We recall that the notions of strong measurability and weak measurability are equivalent in a separable Banach space, and so functions will be referred to as measurable, without ambiguity. 
The next theorem will be used frequently. Its proof is a consequence of the Pettis measurability theorem (see [4, Corollary II.1.3]).

TheOREM 2.1. Let $(K, \mathcal{F})$ be a measurable space and $X$ a separable $B a$ nach space. If $f: K \rightarrow X$ is a measurable function, then there exists a sequence of countably valued measurable functions converging uniformly to $f$.

Denote by $L^{\infty}(K ; X)$ the set of all $\mathcal{F}$-measurable bounded functions from $K$ into $X$. For every $f \in L^{\infty}(K ; X)$ let

$$
\|f\|_{\infty}=\sup _{x \in K}\|f(x)\|_{X} .
$$

If $f \in L^{\infty}(K ; X)$, then the uniformly convergent sequence $f_{n}$ in Theorem 2.1 may be chosen so that $\left\|f_{n}\right\|_{\infty} \leq 2\|f\|_{\infty}$ for all $n \in \mathbb{N}$.

Definition 2.2. Let $Y$ be a Banach space and $(K, \mathcal{F})$ a measurable space. A $Y$-valued measure on $\mathcal{F}$ (or $Y$-measure) is a countably additive set function on $\mathcal{F}$ which takes values in the Banach space $Y$. The (total) variation of the $Y$-valued measure $\nu$ is

$$
|\nu|(A)=\sup \left\{\sum_{j=1}^{N}\left\|\nu\left(A_{j}\right)\right\|_{Y}:\left(A_{j}\right)_{j=1}^{N} \in \pi_{A}\right\}, \quad A \in \mathcal{F} .
$$

That $|\nu|$ is a positive, countably additive measure on $\mathcal{F}$ follows from standard arguments (e.g. [13, Theorem 6.2]). As is customary, we denote $|\nu|(K)$ by $\|\nu\|_{M}$.

Theorem 2.3 (Pettis). Let $Y$ be a Banach space with dual space $Y^{*}$. A $Y$-valued measure $\nu$ is countably additive (in the norm topology) if $y^{*} \nu$ is a countably additive scalar-valued measure for all $y^{*} \in Y^{*}$ (in which case $\nu$ is said to be weakly countably additive).

This theorem had been proved first for normed spaces by Pettis in [12], and was later extended to locally convex spaces by Grothendieck in [8].

Let $X, Y$, and $Z$ be Banach spaces and suppose there exists a bilinear map $\langle\cdot, \cdot\rangle$ on $X \times Y$ taking values in $Z$ such that

$$
\|\langle x, y\rangle\|_{Z} \leq\|x\|_{X}\|y\|_{Y}
$$

for all $(x, y) \in X \times Y$. Suppose $(K, \mathcal{F})$ is a measurable space. Let $\nu$ be a countably additive $Y$-valued measure on $\mathcal{F}$ with finite total variation. Let $f \in L^{\infty}(K ; X)$ be countably valued, say

$$
f=\sum_{j=1}^{\infty} x_{j} \mathbb{1}_{A_{j}},
$$

where $\left(A_{j}\right)$ is a sequence of pairwise disjoint measurable sets and $\left(x_{j}\right)$ is a 
bounded sequence in $X$. Define the integral of $f$ with respect to $\nu$ by

$$
\int_{X}\langle f(x), \nu(d x)\rangle:=\sum_{j=1}^{\infty}\left\langle x_{j}, \nu\left(A_{j}\right)\right\rangle .
$$

The sum defining the integral in (2.2) converges (in norm) absolutely because $f$ is bounded and $\nu$ has finite variation.

For $f \in L^{\infty}(K ; X)$, define the integral of $f$ with respect to $\nu$ by

$$
\int_{X}\langle f(x), \nu(d x)\rangle:=\lim _{n \rightarrow \infty} \int_{X}\left\langle f_{n}(x), \nu(d x)\right\rangle,
$$

where $\left(f_{n}\right)$ is a sequence of countably valued measurable functions such that $f_{n} \rightarrow f$ uniformly and $\left\|f_{n}\right\|_{\infty} \leq 2\|f\|_{\infty}$ for all $n$. Such a sequence exists by Theorem 2.1, provided $X$ is separable.

Theorem 2.4. Let $X, Y$, and $Z$ be Banach spaces with $X$ separable, and suppose $\langle\cdot, \cdot\rangle$ is a bounded bilinear map on $X \times Y$ taking values in $Z$. Let $(K, \mathcal{F})$ be a measurable space, and let $\nu$ be a countably additive $Y$-valued measure on $\mathcal{F}$ with finite total variation. If $f \in L^{\infty}(K ; X)$, then the integral of $f$ with respect to $\nu$ given by (2.3) is well-defined, linear in $f$, and

$$
\left\|\int_{X}\langle f(x), \nu(d x)\rangle\right\|_{Z} \leq\|f\|_{\infty}\|\nu\|_{M} .
$$

Proof. Let $f \in L^{\infty}(K ; X)$ be countably valued as in (2.1). The integral is independent of the representation used for $f$ because of the countable additivity of $\nu$. For any $N \in \mathbb{N}$,

$$
\begin{aligned}
\sum_{j=1}^{N}\left\|\left\langle x_{j}, \nu\left(A_{j}\right)\right\rangle\right\|_{Z} & \leq \sum_{j=1}^{N}\left\|x_{j}\right\|_{X}\left\|\nu\left(A_{j}\right)\right\|_{Y} \leq\left(\sup _{j}\left\|x_{j}\right\|_{X}\right) \sum_{j=1}^{N}\left\|\nu\left(A_{j}\right)\right\|_{Y} \\
& \leq\|f\|_{\infty}\|\nu\|_{M} .
\end{aligned}
$$

Taking the limit as $N \rightarrow \infty$ provides the desired bound. The linearity of the integral follows from the fact that the series $\sum_{j}\left\langle x_{j}, \nu\left(A_{j}\right)\right\rangle$ converges absolutely. To prove the theorem for functions in $L^{\infty}(K ; X)$, use Theorem 2.1 and the definition in $(2.3)$. The integral is independent of the defining sequence by uniform convergence.

When the domain is apparent, we denote $\int_{X}\langle f(x), \nu(d x)\rangle$ by $\int\langle f, d \nu\rangle$.

Remark 2.5. Assume $X, Y, Z,(K, \mathcal{F})$, and $\nu$ are as in Theorem 2.4. To compare (2.3) with the integral of Dinculeanu (e.g., [5]), let $L(X, Z)$ denote the set of bounded linear operators from $X$ into $Z$, and define a measure $m: \mathcal{F} \rightarrow L(X, Z)$ by

$$
m(A)(x)=\langle x, \nu(A)\rangle, \quad A \in \mathcal{F},
$$


for all $x \in X$. Then for any $f \in L^{\infty}(K ; X)$,

$$
\int\langle f, d \nu\rangle=\int f d m
$$

where the integral on the right is Dinculeanu's integral.

Remark 2.6. In Theorem 2.4, the separability of the Banach space $X$ was used to ensure the existence of uniformly approximating sequences. Strongly measurable functions are separably valued (see the Pettis measurability theorem, e.g., Theorem II.1.2 in [4]), and so they can be integrated as in Theorem 2.4, even when $X$ is not separable.

Remark 2.7. Suppose $K$ is a compact Hausdorff space. Denote by $C(K ; X)$ the set of continuous functions on $K$ taking values in the Banach space $X$. Functions in $C(K ; X)$ can be approximated uniformly by simple functions. (The proof is the same as in the scalar case, with norms replacing absolute values.) Consequently, functions in $C(K ; X)$ are strongly measurable.

3. The iterated integral. Suppose $(X, \mathcal{A})$ and $(Y, \mathcal{B})$ are measurable spaces. For any $f \otimes g \in L^{\infty}(X) \otimes L^{\infty}(Y)$ the integral with respect to $\mu \in$ $F_{2}(\mathcal{A}, \mathcal{B})$ can be evaluated iteratively, that is, $\int f \otimes g d \mu=\int f d \mu_{g}$ (see 1.8$)$. Now consider bounded measurable functions $f: X \rightarrow H, g: Y \rightarrow H$, where $H$ is a separable Hilbert space. Let

$$
\langle f, g\rangle(x, y)=\langle f(x), g(y)\rangle, \quad(x, y) \in X \times Y .
$$

We construct an iterated integral $\int\left\langle f, d \nu_{g}\right\rangle$, where $\nu_{g}$ is a Hilbert space-valued measure, and show

$$
\int\langle f, g\rangle d \mu=\int\left\langle f, d \nu_{g}\right\rangle,
$$

where $\int\langle f, g\rangle d \mu$ is given by 1.4 .

\subsection{An extension of the Lindenstrauss and Pełczyński inequal-} ity. In order to continue, we extend Theorem 1.1 to infinite sums. We begin by recalling the following Fubini-type property for $\beta \in F_{2}(\mathbb{N}, \mathbb{N})$, which is taken from Corollary IV.7 in [2]. and

Lemma 3.1 (Blei). Let $\beta \in F_{2}(\mathbb{N}, \mathbb{N})$. Then $\sum_{n=1}^{\infty} \sum_{m=1}^{\infty} \beta(m, n)$ exists

$$
\sum_{n=1}^{\infty} \sum_{m=1}^{\infty} \beta(m, n)=\sum_{m=1}^{\infty} \sum_{n=1}^{\infty} \beta(m, n) .
$$

This lemma, which remains true in higher dimensions, leads directly to the following: 
Lemma 3.2. Suppose $\left(a_{i j}\right) \in F_{2}(\mathbb{N}, \mathbb{N})$. If $\left(x_{i}\right)$ and $\left(y_{j}\right)$ are bounded sequences in a Hilbert space, then the series $\sum_{i} \sum_{j} a_{i j}\left\langle x_{i}, y_{j}\right\rangle$ converges and

$$
\sum_{i=1}^{\infty} \sum_{j=1}^{\infty} a_{i j}\left\langle x_{i}, y_{j}\right\rangle=\sum_{j=1}^{\infty} \sum_{i=1}^{\infty} a_{i j}\left\langle x_{i}, y_{j}\right\rangle
$$

Moreover,

$$
\left|\sum_{i=1}^{\infty} \sum_{j=1}^{\infty} a_{i j}\left\langle x_{i}, y_{j}\right\rangle\right| \leq K_{G}\|a\|_{F_{2}}\left(\sup _{i \in \mathbb{N}}\left\|x_{i}\right\|\right)\left(\sup _{j \in \mathbb{N}}\left\|y_{j}\right\|\right),
$$

and

$$
\sum_{i=1}^{\infty}\left\|\sum_{j=1}^{\infty} y_{j} a_{i j}\right\| \leq K_{G}\|a\|_{F_{2}}\left(\sup _{j \in \mathbb{N}}\left\|y_{j}\right\|\right) .
$$

Proof. Let $\beta(i, j)=a_{i j}\left\langle x_{i}, y_{j}\right\rangle$. By Lemma 3.1, it suffices to show $\beta \in$ $F_{2}(\mathbb{N}, \mathbb{N})$. Let $N \in \mathbb{N}$ and let $\left|t_{i}\right|,\left|s_{j}\right| \leq 1$ for $i, j=1, \ldots, N$. Then

$$
\left|\sum_{i, j=1}^{N} \beta(i, j) t_{i} s_{j}\right|=\left|\sum_{i, j=1}^{N} a_{i j}\left\langle x_{i}, y_{j}\right\rangle t_{i} s_{j}\right|=\left|\sum_{i, j=1}^{N} a_{i j}\left\langle t_{i} x_{i}, s_{j} y_{j}\right\rangle\right| .
$$

By Theorem 1.1, this is bounded by

$$
K_{G}\|a\|_{F_{2}}\left(\sup _{i \in \mathbb{N}}\left\|x_{i}\right\|\right)\left(\sup _{j \in \mathbb{N}}\left\|y_{j}\right\|\right),
$$

which is finite, by assumption. Thus, $\beta \in F_{2}(\mathbb{N}, \mathbb{N})$, as required.

The inequality in 3.2 follows by taking limits. The inequality in 3.3 . is a restatement of (3.2) with $x_{i}$ the element of norm 1 in $H$ such that

$$
\left\langle x_{i}, \sum_{j=1}^{\infty} y_{j} a_{i j}\right\rangle=\left\|\sum_{j=1}^{\infty} y_{j} a_{i j}\right\|, \quad i \in \mathbb{N} .
$$

This final sum is absolutely convergent because $\left(a_{i j}\right) \in F_{2}(\mathbb{N}, \mathbb{N})$ :

$$
\sum_{j=1}^{N}\left\|y_{j} a_{i j}\right\|=\sum_{j=1}^{N}\left\|y_{j}\right\| \epsilon_{j} a_{i j} \leq\|a\|_{F_{2}} \sup _{j \in \mathbb{N}}\left\|y_{j}\right\|,
$$

where $\epsilon_{j}=\operatorname{sgn}\left(a_{i j}\right)(i$ is fixed $)$.

3.2. Constructing an iterated integral. Let $H$ be a separable Hilbert space and let $(X, \mathcal{A})$ and $(Y, \mathcal{B})$ be measurable spaces. For any $g \in L^{\infty}(Y ; H)$ and $\mu \in F_{2}(\mathcal{A}, \mathcal{B})$, define an $H$-valued set function on $\mathcal{A}$ by

$$
\nu_{g}(A):=\int_{Y} g(y) \mu(A, d y), \quad A \in \mathcal{A} .
$$

For each $A \in \mathcal{A}$, the set function $\mu(A, \cdot)$ is a scalar-valued measure of finite total variation, so the integral is well-defined by Theorem 2.4, with 
both $X$ and $Z$ taken to be $H, Y$ taken to be the scalar field, and the bilinear map taken to be multiplication.

Lemma 3.3. Let $E \in \mathcal{A}$. Then $\nu_{g}(E)$ is the unique element of $H$ with the property

$$
\left\langle x, \nu_{g}(E)\right\rangle=\int_{Y}\langle x, g(y)\rangle \mu(E, d y), \quad x \in H .
$$

Proof. The right-hand side of (3.4) is well-defined, because $\langle x, g\rangle$ is a bounded measurable scalar-valued function for each $x \in H$. It follows that, for fixed $g \in L^{\infty}(Y ; H), \mu \in F_{2}(\mathcal{A}, \mathcal{B})$, and $E \in \mathcal{A}$,

$$
\Lambda(x):=\int_{Y}\langle x, g(y)\rangle \mu(E, d y), \quad x \in H,
$$

is a bounded linear functional on $H$. Therefore, there exists a unique element $z \in H$ such that

$$
\langle x, z\rangle=\Lambda(x), \quad x \in H .
$$

It only remains to show $z=\nu_{g}(E)$.

If $g=\sum_{j=1}^{\infty} b_{j} \mathbb{1}_{B_{j}}$, where $\left(B_{j}\right)$ is a sequence of pairwise disjoint sets in $\mathcal{B}$ and $\left(b_{j}\right)$ is a bounded sequence in $H$, then, by 2.2$)$, for each $x \in H$,

$$
\left\langle x, \nu_{g}(E)\right\rangle=\left\langle x, \sum_{j=1}^{\infty} b_{j} \mu\left(E, B_{j}\right)\right\rangle=\sum_{j=1}^{\infty}\left\langle x, b_{j}\right\rangle \mu\left(E, B_{j}\right),
$$

where convergence follows from Lemma 3.2 (the Grothendieck inequality). Since

$$
\int_{Y}\langle x, g(y)\rangle \mu(E, d y)=\sum_{j=1}^{\infty}\left\langle x, b_{j}\right\rangle \mu\left(E, B_{j}\right),
$$

the claim is proved in this case. (Recall that for $E \in \mathcal{A}, \mu(E, \cdot)$ is a measure on $\mathcal{B}$.)

For $g \in L^{\infty}(Y ; H)$, let $\left(g_{n}\right)$ be a sequence of countably valued bounded measurable functions converging uniformly to $g$. By definition,

$$
\nu_{g}(E)=\int_{Y} g(y) \mu(E, d y)=\lim _{n \rightarrow \infty} \int_{Y} g_{n}(y) \mu(E, d y)=\lim _{n \rightarrow \infty} \nu_{g_{n}}(E) .
$$

For each $n$, the function $g_{n}$ is countably valued, hence the lemma holds for each $\nu_{n}$. Therefore, for all $x \in H$,

$$
\left\langle x, \nu_{g}(E)\right\rangle=\lim _{n \rightarrow \infty}\left\langle x, \nu_{g_{n}}(E)\right\rangle=\lim _{n \rightarrow \infty} \int_{Y}\left\langle x, g_{n}(y)\right\rangle \mu(E, d y) .
$$

For a fixed set $E, \mu(E, \cdot)$ is a measure of finite total variation. Therefore, by 
the bounded convergence theorem,

$$
\lim _{n \rightarrow \infty} \int_{Y}\left\langle x, g_{n}(y)\right\rangle \mu(E, d y)=\int_{Y}\langle x, g(y)\rangle \mu(E, d y),
$$

which provides the desired result.

THEOREM 3.4. For $g \in L^{\infty}(Y ; H)$, the $H$-valued set function $\nu_{g}$ defined in (3.4) is an $H$-valued countably additive measure.

Proof. We will show the measure is weakly countably additive and the result will follow by Theorem 2.3. Let $\left(A_{i}\right)_{j=1}^{\infty}$ be a sequence of pairwise disjoint measurable sets, and let $A=\bigcup_{j=1}^{\infty} A_{j}$. Let $x \in H$. By Lemma 3.3 .

$$
\left\langle x, \nu_{g}(A)\right\rangle=\int_{Y}\langle x, g(y)\rangle \mu(A, d y)=\mu_{\langle x, g\rangle}(A) .
$$

Here, $\langle x, g\rangle(y)=\langle x, g(y)\rangle, y \in Y$, is a scalar-valued, bounded measurable function, and so $\mu_{\langle x, g\rangle}$ is the scalar-valued set function defined in 1.9$)$. Then $\mu_{\langle x, g\rangle}$ is a measure, and so

$$
\mu_{\langle x, g\rangle}\left(\bigcup_{i=1}^{\infty} A_{i}\right)=\sum_{i=1}^{\infty} \mu_{\langle x, g\rangle}\left(A_{i}\right)=\sum_{i=1}^{\infty} \int_{Y}\langle x, g(y)\rangle \mu\left(A_{i}, d y\right) .
$$

Once again applying Lemma 3.3 , we have

$$
\left\langle x, \nu_{g}(A)\right\rangle=\sum_{i=1}^{\infty}\left\langle x, \nu_{g}\left(A_{i}\right)\right\rangle
$$

and $\nu_{g}$ is weakly countably additive, as required.

Theorem 3.5. Let $g \in L^{\infty}(Y ; H)$. Then $\nu_{g}$ has finite total variation and

$$
\left\|\nu_{g}\right\|_{M} \leq K_{G}\|\mu\|_{F_{2}}\|g\|_{\infty}
$$

where $K_{G}$ is the Grothendieck constant.

Proof. Let $\left(A_{i}\right)_{i=1}^{N}$ be an arbitrary finite partition of the set $A \in \mathcal{A}$. First, suppose $g=\sum_{j=1}^{\infty} b_{j} \mathbb{1}_{B_{j}}$ is a countably valued bounded measurable function. By (3.3) from Lemma 3.2.

$$
\begin{aligned}
\sum_{i=1}^{N}\left\|\nu_{g}\left(A_{i}\right)\right\| & =\sum_{i=1}^{N}\left\|\sum_{j=1}^{\infty} b_{j} \mu\left(A_{i}, B_{j}\right)\right\| \leq K_{G}\|\mu\|_{F_{2}}\left(\sup _{j \in \mathbb{N}}\left\|y_{j}\right\|\right) \\
& \leq K_{G}\|\mu\|_{F_{2}}\|g\|_{\infty} .
\end{aligned}
$$

Now suppose $g \in L^{\infty}(Y ; H)$. By Theorem 2.1, there exists a sequence $\left(g_{n}\right)$ of countably valued measurable functions converging uniformly to $g$. Then

$$
\sum_{i=1}^{N}\left\|\nu_{g}\left(A_{i}\right)\right\|=\lim _{n \rightarrow \infty} \sum_{i=1}^{N}\left\|\nu_{g_{n}}\left(A_{i}\right)\right\| .
$$


By (3.6),

$$
\sum_{i=1}^{N}\left\|\nu_{g_{n}}\left(A_{i}\right)\right\| \leq K_{G}\|\mu\|_{F_{2}}\left\|g_{n}\right\|_{\infty} .
$$

The result follows by taking the supremum over partitions of $A$.

Theorem 3.6. Let $(X, \mathcal{A})$ and $(Y, \mathcal{B})$ be measurable spaces and let $H$ be a separable Hilbert space with inner product $\langle\cdot, \cdot\rangle$. For $f \in L^{\infty}(X ; H)$ and $g \in L^{\infty}(Y ; H)$, the $H$-valued function $f$ is integrable with respect to the $H$-valued measure $\nu_{g}$, and

$$
\left|\int\left\langle f, d \nu_{g}\right\rangle\right| \leq\|f\|_{\infty}\left\|\nu_{g}\right\|_{M} \leq K_{G}\|\mu\|_{F_{2}}\|f\|_{\infty}\|g\|_{\infty} .
$$

Proof. This follows from Theorems 2.4 and 3.5 .

Lemma 3.7. Let $g, h \in L^{\infty}(Y ; H)$. Then

$$
\nu_{g}(A)-\nu_{h}(A)=\nu_{g-h}(A), \quad A \in \mathcal{A} .
$$

Proof. This follows from the definition of the integral in (2.3). For countably valued functions, the result follows from the absolute convergence of the defining sums (see the proof of Theorem 2.4). For the general case, take limits.

Lemma 3.8. Let $f \in L^{\infty}(X ; H)$ and $g \in L^{\infty}(Y ; H)$. If $\left(f_{n}\right)$ and $\left(g_{n}\right)$ are sequences of bounded measurable functions converging uniformly (in norm) to $f$ and $g$, respectively, then

$$
\int\left\langle f, d \nu_{g}\right\rangle=\lim _{n \rightarrow \infty} \int\left\langle f_{n}, d \nu_{g_{n}}\right\rangle .
$$

Proof. By Theorem 2.4 and Lemma 3.7.

$$
\left|\int\left\langle f, d \nu_{g}\right\rangle-\int\left\langle f_{n}, d \nu_{g_{n}}\right\rangle\right| \leq\left|\int\left\langle f-f_{n}, d \nu_{g}\right\rangle\right|+\left|\int\left\langle f_{n}, d \nu_{g-g_{n}}\right\rangle\right| .
$$

By Theorem 3.6, this is bounded by

$$
K_{G}\|\mu\|_{F_{2}}\left(\left\|f-f_{n}\right\|_{\infty}\|g\|_{\infty}+\left\|f_{n}\right\|_{\infty}\left\|g-g_{n}\right\|_{\infty}\right) .
$$

For sufficiently large $n$, this is arbitrarily small. The result follows.

Lemma 3.9. Let $f \in L^{\infty}(X ; H)$ and $g \in L^{\infty}(Y ; H)$. Suppose $\left(f_{n}\right)$ and $\left(g_{n}\right)$ are sequences of bounded measurable functions converging uniformly (in norm) to $f$ and $g$, respectively. Then for all $\mu \in F_{2}(\mathcal{A}, \mathcal{B})$,

$$
\int\langle f, g\rangle d \mu=\lim _{n \rightarrow \infty} \int\left\langle f_{n}, g_{n}\right\rangle d \mu,
$$

where $\int\langle f, g\rangle d \mu$ is given by 1.4 .

Proof. Since

$$
\int\langle f, g\rangle d \mu-\int\left\langle f_{n}, g_{n}\right\rangle d \mu=\int\left\langle f-f_{n}, g\right\rangle d \mu+\int\left\langle f_{n}, g-g_{n}\right\rangle d \mu
$$

the result follows from Theorem 1.2 and uniform convergence. 
Theorem 3.10. Let $(X, \mathcal{A})$ and $(Y, \mathcal{B})$ be measurable spaces and $H$ a separable Hilbert space with inner product $\langle\cdot, \cdot\rangle$. Let $f \in L^{\infty}(X ; H)$ and $g \in$ $L^{\infty}(Y ; H)$. If $\mu \in F_{2}(\mathcal{A}, \mathcal{B})$, then

$$
\int\langle f, g\rangle d \mu=\int\left\langle f, d \nu_{g}\right\rangle,
$$

where $\int\langle f, g\rangle d \mu$ is given by (1.4).

Proof. In light of Lemmas 3.8 and 3.9 , it suffices to show (3.7) for countably valued bounded measurable functions.

Let $f=\sum_{j=1}^{\infty} \boldsymbol{a}^{(j)} \mathbb{1}_{A_{j}}$ and $g=\sum_{k=1}^{\infty} \boldsymbol{b}^{(k)} \mathbb{1}_{B_{k}}$, where $\left(A_{j}\right)$ and $\left(B_{k}\right)$ are sequences of pairwise disjoint measurable sets, and $\left\|\boldsymbol{a}^{(j)}\right\|_{H} \leq 1,\left\|\boldsymbol{b}^{(k)}\right\|_{H} \leq 1$. Suppose $H$ has basis $\left(e_{j}\right)$. For each $j, k \in \mathbb{N}$, let $a_{n}^{(j)}$ and $b_{n}^{(k)}$ be scalar sequences such that

$$
\boldsymbol{a}^{(j)}=\sum_{n=1}^{\infty} a_{n}^{(j)} e_{n}, \quad \boldsymbol{b}^{(k)}=\sum_{n=1}^{\infty} b_{n}^{(k)} e_{n} .
$$

Then

$$
f=\sum_{n=1}^{\infty}\left(\sum_{j=1}^{\infty} a_{n}^{(j)} \mathbb{1}_{A_{j}}\right) e_{n} \quad \text { and } \quad g=\sum_{n=1}^{\infty}\left(\sum_{k=1}^{\infty} b_{n}^{(k)} \mathbb{1}_{B_{k}}\right) e_{n},
$$

and so the coordinate functions are

$$
f_{n}=\sum_{j=1}^{\infty} a_{n}^{(j)} \mathbb{1}_{A_{j}} \quad \text { and } \quad g_{n}=\sum_{k=1}^{\infty} b_{n}^{(k)} \mathbb{1}_{B_{k}} .
$$

Observe that

$$
\begin{aligned}
\int\left\langle f, \nu_{g}\right\rangle d \mu & =\sum_{j, k=1}^{\infty}\left\langle\boldsymbol{a}^{(j)}, \boldsymbol{b}^{(k)}\right\rangle \mu\left(A_{j}, B_{k}\right) \\
& =\sum_{j, k=1}^{\infty}\left(\sum_{n=1}^{\infty} a_{n}^{(j)} b_{n}^{(k)}\right) \mu\left(A_{j}, B_{k}\right) .
\end{aligned}
$$

Furthermore,

$$
\begin{aligned}
\int f_{n} \otimes g_{n} d \mu & =\int\left(\sum_{j, k=1}^{\infty} a_{n}^{(j)} b_{n}^{(k)} \mathbb{1}_{A_{j}} \otimes \mathbb{1}_{B_{k}}\right) d \mu \\
& =\sum_{j, k=1}^{\infty} a_{n}^{(j)} b_{n}^{(k)} \mu\left(A_{j}, B_{k}\right) .
\end{aligned}
$$

Therefore, (3.7) will follow from (3.8) and (3.9), provided we can interchange the order of summation in (3.8). To that end, let

$$
\lambda(j, k, n)=a_{n}^{(j)} b_{n}^{(k)} \mu\left(A_{j}, B_{k}\right), \quad j, k, n \in \mathbb{N} .
$$

It will suffice to show $\lambda \in F_{3}(\mathbb{N}, \mathbb{N}, \mathbb{N}$ ) (see Lemma 3.2 ). 
Let $N \in \mathbb{N}$ be arbitrary and let $\epsilon_{j}, \delta_{k}, \gamma_{n}$ be arbitrary choices of \pm 1 for each $j, k, n \in \mathbb{N}$. Then

$$
\sum_{j, k, n=1}^{N} \lambda(j, k, n) \epsilon_{j} \delta_{k} \gamma_{n}=\sum_{j, k=1}^{N}\left(\sum_{n}^{N} a_{n}^{(j)} b_{n}^{(k)} \gamma_{n}\right) \mu\left(A_{j}, B_{k}\right) \epsilon_{j} \delta_{k} .
$$

Let

$$
\boldsymbol{a}^{(j, N)}=\sum_{n=1}^{N} a_{n}^{(j)} e_{n} \quad \text { and } \quad \boldsymbol{b}^{(k, N, \gamma)}=\sum_{n=1}^{N} b_{n}^{(k)} \gamma_{n} e_{n} .
$$

Then (3.10) becomes

$$
\begin{aligned}
\sum_{j, k, n=1}^{N} \lambda(j, k, n) \epsilon_{j} \delta_{k} \gamma_{n} & =\sum_{j, k=1}^{N}\left\langle\boldsymbol{a}^{(j, N)}, \boldsymbol{b}^{(k, N, \gamma)}\right\rangle \mu\left(A_{j}, B_{k}\right) \epsilon_{j} \delta_{k} \\
& =\sum_{j, k=1}^{N}\left\langle\boldsymbol{a}^{(j, N)} \epsilon_{j}, \boldsymbol{b}^{(k, N, \gamma)} \delta_{k}\right\rangle \mu\left(A_{j}, B_{k}\right) .
\end{aligned}
$$

For each $j, k \in \mathbb{N},\left\|\boldsymbol{a}^{(j, N)} \epsilon_{j}\right\|_{H} \leq\left\|\boldsymbol{a}^{(j)}\right\|_{H} \leq 1,\left\|\boldsymbol{b}^{(k, N, \gamma)} \delta_{k}\right\|_{H} \leq\left\|\boldsymbol{b}^{(k)}\right\|_{H}$ $\leq 1$. Therefore, by the Grothendieck inequality,

$$
\left|\sum_{j, k, n=1}^{N} \lambda(j, k, n) \epsilon_{j} \delta_{k} \gamma_{n}\right| \leq K_{G}\|\mu\|_{F_{2}} .
$$

It follows that $\lambda \in F_{3}(\mathbb{N}, \mathbb{N}, \mathbb{N})$, as required.

3.3. The order of integration. Let $(X, \mathcal{A})$ and $(Y, \mathcal{B})$ be measurable spaces and $H$ a separable Hilbert space with inner product $\langle\cdot, \cdot\rangle$. When integrating a tensor product of scalar-valued bounded measurable functions, the order of integration could be reversed when evaluating iteratively (see (1.8)). We now show the same is true when integrating an inner product iteratively.

For $f \in L^{\infty}(X, H)$, define an $H$-valued measure on $\mathcal{B}$ by

$$
\nu^{f}(B)=\int_{X} f(x) \mu(d x, B), \quad B \in \mathcal{B} .
$$

The set function $\nu^{f}$ is well-defined by Theorem 2.3 (cf. (3.4)). The proof that $\nu^{f}$ is an $H$-valued measure with the same properties as $\nu_{g}$ is similar to the proofs of Theorems 3.4 and 3.5 . The integral of an $H$-valued function $g$ with respect to the $H$-valued measure $\nu^{f}$ is given by Theorem 2.3 (cf. Theorem 3.6), and the bound is the same.

Theorem 3.11. Let $(X, \mathcal{A})$ and $(Y, \mathcal{B})$ be measurable spaces and $H$ a separable Hilbert space with inner product $\langle\cdot, \cdot\rangle$. If $f \in L^{\infty}(X ; H)$ and $g \in$ 
$L^{\infty}(Y ; H)$, then

$$
\int_{X}\left\langle f(x), \nu_{g}(d x)\right\rangle=\int_{Y}\left\langle g(y), \nu^{f}(d y)\right\rangle .
$$

Proof. It suffices to show the theorem when $f$ and $g$ are countably valued bounded measurable functions. Let

$$
f=\sum_{j=1}^{\infty} a_{j} \mathbb{1}_{A_{j}} \quad \text { and } \quad g=\sum_{k=1}^{\infty} b_{k} \mathbb{1}_{B_{k}},
$$

where $\left(A_{j}\right)$ and $\left(B_{k}\right)$ are sequences of pairwise disjoint sets in $\mathcal{A}$ and $\mathcal{B}$, respectively, and $\left(a_{j}\right),\left(b_{k}\right)$ are bounded sequences in $H$. By Lemma 3.2 .

$$
\begin{aligned}
\int\left\langle f, d \nu_{g}\right\rangle & =\sum_{j=1}^{\infty} \sum_{k=1}^{\infty}\left\langle a_{j}, b_{k}\right\rangle \mu\left(A_{j}, B_{k}\right) \\
& =\sum_{k=1}^{\infty} \sum_{j=1}^{\infty}\left\langle a_{j}, b_{k}\right\rangle \mu\left(A_{j}, B_{k}\right)=\int\left\langle g, d \nu^{f}\right\rangle .
\end{aligned}
$$

3.4. A remark about general Banach spaces. We have proved for measurable spaces $(X, \mathcal{A})$ and $(Y, \mathcal{B})$, and a separable Hilbert space $H$, that any $\mu \in F_{2}(\mathcal{A}, \mathcal{B})$ and $g \in L^{\infty}(Y ; H)$ determine an $H$-valued measure $\nu_{g}=$ $\int_{Y} g(y) \mu(\cdot, d y)$ on $\mathcal{A}$, and $\left\|\nu_{g}\right\|_{M} \leq K_{G}\|\mu\|_{F_{2}}\|g\|_{\infty}$. The same cannot be said for general Banach spaces.

Theorem 3.12. Let $E$ be a separable Banach space with dual $E^{*}$. Suppose $(Y, \mathcal{F})$ is a measurable space such that $\mathcal{F}$ is infinite. For all $\mu \in F_{2}(\mathcal{F}, \mathcal{F})$ and $g \in L^{\infty}(Y ; E)$, if the E-valued set function

$$
\nu_{g}=\int_{Y} g(y) \mu(\cdot, d y)
$$

is an E-valued measure on $\mathcal{F}$ such that $\left\|\nu_{g}\right\|_{M} \leq K\|\mu\|_{F_{2}}\|g\|_{\infty}$ for some universal constant $K$, then $E$ is a Hilbert space.

Proof. The proof follows from Corollary 1 of Theorem 4.2 in [10]:

Proposition 3.13 (Lindenstrauss and Pełczyński). Let E be a Banach space for which there is a constant $K$ such that the following is true: Whenever $\left(a_{j k}\right) \in F_{2}(\mathbb{N}, \mathbb{N})$ with $\|a\|_{F_{2}} \leq 1$, then

$$
\left|\sum_{j, k} a_{j k}\left\langle v_{j}, v_{k}^{*}\right\rangle\right| \leq K \sup _{j}\left\|v_{j}\right\| \sup _{k}\left\|v_{k}^{*}\right\|
$$

for every choice of $\left(v_{j}\right)_{j=1}^{N} \subset E$ and $\left(v_{k}^{*}\right)_{k=1}^{N} \subset E^{*}$. Then $E$ is isomorphic to a Hilbert space.

To prove Theorem 3.12 , let $\left(a_{j k}\right) \in F_{2}(\mathbb{N}, \mathbb{N})$ with $\|a\|_{F_{2}} \leq 1$. Let $\left(A_{j}\right)_{j=1}^{\infty}$ be a sequence of nonempty pairwise disjoint sets in $\mathcal{F}$, and for each $j \in \mathbb{N}$, 
choose $x_{j} \in A_{j}$. Define a scalar-valued bimeasure

$$
\mu=\sum_{j, k=1}^{n} a_{j k} \delta_{x_{j}} \otimes \delta_{x_{k}}
$$

where $\delta_{x}$ is the Dirac measure at $x \in Y$. Let $v_{1}, \ldots, v_{n}$ be arbitrary vectors in the unit ball of $E$ and consider the $E$-valued simple measurable function $g=\sum_{k=1}^{n} v_{k} \mathbb{1}_{A_{k}}$. By duality,

$$
\begin{aligned}
\sum_{j=1}^{n}\left\|\nu_{g}\left(A_{j}\right)\right\|_{E} & =\sup \left\{\left|\sum_{j=1}^{n}\left\langle v_{j}^{*}, \nu_{g}\left(A_{j}\right)\right\rangle\right|:\left\|v_{j}^{*}\right\|_{E^{*}} \leq 1\right\} \\
& =\sup \left\{\left|\sum_{j=1}^{n} \sum_{k=1}^{n}\left\langle v_{j}^{*}, v_{k}\right\rangle \mu\left(A_{j}, A_{k}\right)\right|:\left\|v_{j}^{*}\right\|_{E^{*}} \leq 1\right\} .
\end{aligned}
$$

Therefore, for all $\left\|v_{j}^{*}\right\|_{E^{*}} \leq 1$,

$$
\left|\sum_{j=1}^{n} \sum_{k=1}^{n}\left\langle v_{j}^{*}, v_{k}\right\rangle \mu\left(A_{j}, A_{k}\right)\right| \leq\left|\nu_{g}\right|(E),
$$

which, by assumption, is bounded by $K$. Therefore, by the definition of $\mu$,

$$
\left|\sum_{j=1}^{n} \sum_{k=1}^{n}\left\langle v_{j}^{*}, v_{k}\right\rangle a_{j k}\right| \leq K .
$$

The choices of $\left\|v_{k}\right\|_{E} \leq 1$ and $\left\|v_{j}^{*}\right\|_{E^{*}} \leq 1$ were arbitrary. By Proposition 3.13, this condition can be satisfied only if $E$ is a Hilbert space.

3.5. Extension to higher dimensions. In dimensions greater than two, not all multilinear functionals satisfy Grothendieck-type inequalities. We distinguish those that do in the following definition (see [2, Chapter VIII]).

Definition 3.14. Let $A$ be an $n$-linear functional on a Hilbert space $H$. Let

$$
\|A\|_{p b_{n}}=\sup \left\{\left\|\left.A\right|_{E_{1} \times \cdots \times E_{n}}\right\|_{\mathcal{V}_{n}\left(E_{1}, \ldots, E_{n}\right)}: E_{j} \subset B_{H},\left|E_{j}\right|<\infty, j=1, \ldots, n\right\},
$$

where $B_{H}$ is the unit ball in $H$, and $\mathcal{V}_{n}\left(E_{1}, \ldots, E_{n}\right)=L^{\infty}\left(E_{1}\right) \hat{\otimes} \cdots \hat{\otimes}$ $L^{\infty}\left(E_{n}\right)$. If $\|A\|_{p b_{n}}<\infty$, then $A$ is said to be projectively bounded. Otherwise, $A$ is said to be projectively unbounded.

The results of this paper can be generalized to higher dimensions for multilinear functionals that are projectively bounded. The arguments are similar to the two-dimensional case, and will not be included here.

TheOREM 3.15. Let $A$ be a projectively bounded n-linear functional on a separable Hilbert space $H$. Let $\left(K_{i}, \mathcal{F}_{i}\right)$ be a measurable space, and let $f_{i} \in$ 
$L^{\infty}\left(K_{i} ; H\right), i=1, \ldots, n$. Let $\mu \in F_{n}\left(\mathcal{F}_{1}, \ldots, \mathcal{F}_{n}\right)$. Let $\nu$ be a function on $\mathcal{F}_{1}$ defined so that, for each $E \in \mathcal{F}_{1}, \nu(E)$ satisfies

$$
\langle\boldsymbol{x}, \nu(E)\rangle=\int_{K_{2} \times \cdots \times K_{n}} A\left(\boldsymbol{x}, f_{2}\left(x_{2}\right), \ldots, f_{n}\left(x_{n}\right)\right) \mu\left(E, d x_{2}, \ldots, d x_{n}\right)
$$

for all $\boldsymbol{x} \in H$. Then $\nu$ is a countably additive, $H$-valued measure of finite total variation, and

$$
\|\nu\|_{M} \leq\|A\|_{p b_{n}}\|\mu\|_{F_{n}}\left\|f_{2}\right\|_{\infty} \cdots\left\|f_{n}\right\|_{\infty} .
$$

Furthermore, $A\left(f_{1}, \ldots, f_{n}\right)$ is integrable with respect to $\mu$ and

$$
\int A\left(f_{1}, \ldots, f_{n}\right) d \mu=\int\left\langle f_{1}, d \nu\right\rangle .
$$

REMARK 3.16. The Grothendieck inequality implies that all bounded bilinear functionals on a Hilbert space are projectively bounded. In dimensions higher than two, however, there exist bounded multilinear functionals on a Hilbert space which are projectively unbounded (see [2, Chapter VIII]). Countably additive measures can be constructed as in (3.13) using projectively unbounded multilinear functionals; however, such measures will necessarily have infinite total variation.

4. An application: an $L^{p}-L^{q}$ Grothendieck-type inequality. Let $(K, \mathcal{F}, \lambda)$ be a measure space, where $\lambda$ is a positive measure. Let $H$ be a separable Hilbert space with inner product $\langle\cdot, \cdot\rangle_{H}$ and norm $\|\cdot\|_{H}$. Let $p$ and $q$ be conjugate exponents, and let $\langle\cdot, \cdot\rangle_{q, p}$ denote the dual action between an $L^{q}$ space (the element on the left) and an $L^{p}$ space (the element on the right). Elements of the Hilbert space $H$, and functions taking values in $H$, will be printed in boldface.

For all $1 \leq p<\infty$ denote by $L^{p}(K, \lambda ; H)$ the space of all (equivalence classes of) measurable functions $\boldsymbol{f}$ taking values in $H$ such that

$$
\int_{K}\|\boldsymbol{f}(x)\|_{H}^{p} \lambda(d x)<\infty .
$$

Equipped with the norm

$$
\|\boldsymbol{f}\|_{L^{p}}=\left(\int_{K}\|\boldsymbol{f}(x)\|_{H}^{p} \lambda(d x)\right)^{1 / p},
$$

the space $L^{p}(K, \lambda ; H)$ forms a Banach space with dual space $L^{q}(K, \lambda ; H)$. Further, simple functions are dense in $L^{p}(K, \lambda ; H)$ ([4, Section IV.1]).

Theorem 4.1. Let $1<p, q<\infty$ be conjugate exponents. Suppose $T$ : $L^{p}(K, \lambda) \rightarrow L^{q}(K, \lambda)$ is a bounded linear map so that

$$
\left|\langle T f, g\rangle_{q, p}\right| \leq 1 \quad \text { for all }\|f\|_{L^{p}},\|g\|_{L^{p}} \leq 1 .
$$


Then $T$ determines a linear map from $L^{p}(K, \lambda ; H)$ to $L^{q}(K, \lambda ; H)$, also denoted by $T$, such that

$$
\left|\langle T \boldsymbol{f}, \boldsymbol{g}\rangle_{q, p}\right| \leq K_{G}\|\boldsymbol{f}\|_{L^{p}}\|\boldsymbol{g}\|_{L^{p}}
$$

for all $\boldsymbol{f}, \boldsymbol{g} \in L^{p}(K, \lambda ; H)$, where $K_{G}$ is the Grothendieck constant.

It was pointed out to us by Nigel Kalton that this theorem is not new. (See Theorem 1.f.14 in [11, p. 93].) We present here an alternative proof, using the techniques developed in the previous sections.

Proof. To extend $T$ to a map from $L^{p}(K, \lambda ; H)$ to $L^{q}(K, \lambda ; H)$, first consider simple functions. If $\left\{A_{1}, \ldots, A_{n}\right\}$ is a collection of pairwise disjoint measurable sets, and $\left\{\boldsymbol{a}_{1}, \ldots, \boldsymbol{a}_{n}\right\}$ is a collection of elements in $H$, then let

$$
T\left(\sum_{j=1}^{n} \boldsymbol{a}_{j} \mathbb{1}_{A_{j}}\right)=\sum_{j=1}^{n} \boldsymbol{a}_{j}\left(T \mathbb{1}_{A_{j}}\right)
$$

This is well-defined by the linearity of $T$. To extend to all functions in $L^{p}(K, \lambda ; H)$, use the continuity of $T$ and the density of simple functions in $L^{p}(K, \lambda ; H)$.

The bilinear map in (4.1) is given by

$$
\langle T \boldsymbol{f}, \boldsymbol{g}\rangle_{q, p}=\int_{K}\langle(T \boldsymbol{f})(y), \boldsymbol{g}(y)\rangle_{H} \lambda(d y) .
$$

Define real-valued functions $f$ and $g$ on $K$ by $f(x)=\|\boldsymbol{f}(x)\|_{H}$ and $g(y)=\|\boldsymbol{g}(y)\|_{H}$ for $x, y \in K$. Without loss of generality, suppose $f, g>0$.

Define a scalar-valued set function $\mu$ by

$$
\mu(E, F)=\left\langle T\left(f \mathbb{1}_{E}\right), g \mathbb{1}_{F}\right\rangle_{q, p}, \quad E, F \in \mathcal{F} .
$$

Since $\|\boldsymbol{f}\|_{L^{p}(K ; H)}=\|f\|_{L^{p}(K)}$ and $\|\boldsymbol{g}\|_{L^{p}(K ; H)}=\|g\|_{L^{p}(K)}$, both $f$ and $g$ are in $L^{p}(K)$, and so $\mu$ is well-defined.

By Theorem 1.2 ,

$$
\left|\int\left\langle\frac{\boldsymbol{f}}{f}, \frac{\boldsymbol{g}}{g}\right\rangle_{H} d \mu\right| \leq K_{G}\|\mu\|_{F_{2}},
$$

provided $\mu \in F_{2}$. Consequently, it will suffice to prove that $\mu \in F_{2}$ with

$$
\|\mu\|_{F_{2}} \leq\|\boldsymbol{f}\|_{L^{p}}\|\boldsymbol{g}\|_{L^{p}}
$$

and

$$
\int_{K \times K}\left\langle\frac{\boldsymbol{f}(x)}{f(x)}, \frac{\boldsymbol{g}(y)}{g(y)}\right\rangle_{H} \mu(d x, d y)=\langle T \boldsymbol{f}, \boldsymbol{g}\rangle_{q, p} .
$$

Without loss of generality, assume $f(x)=g(y)=1$ for all $x \in X$ and $y \in Y$. We show $\mu \in F_{2}$ by showing it is a measure on $\mathcal{F}$ in each argument 
separately. By definition,

$$
\mu(E, F)=\int_{K}\left(T\left(\mathbb{1}_{E}\right)\right)(y)\left(\mathbb{1}_{F}\right)(y) \lambda(d y)
$$

for $E, F \in \mathcal{F}$. Let $E_{0} \in \mathcal{F}$ be fixed. Then

$$
\mu\left(E_{0}, F\right)=\int_{F}\left(T\left(\mathbb{1}_{E_{0}}\right)\right)(y) \lambda(d y),
$$

which is known to be a measure on $\mathcal{F}$ (e.g., Theorem 1.29 in [13]).

Next, fix $F_{0} \in \mathcal{F}$ and let $E=\bigcup_{j} E_{j}$, where $\left(E_{j}\right)$ is a sequence of pairwise disjoint measurable sets. Then

$$
\mu\left(E, F_{0}\right)=\left\langle T\left(\mathbb{1}_{E}\right), \mathbb{1}_{F_{0}}\right\rangle_{q, p} .
$$

By the bounded convergence theorem (for scalar-vaued functions), it follows that $\sum_{j=1}^{N} \mathbb{1}_{E_{j}} \rightarrow \mathbb{1}_{E}$ in $L^{p}(K, \lambda)$ as $N \rightarrow \infty$. By the continuity of $T$,

$$
T\left(\mathbb{1}_{E}\right)=\lim _{N \rightarrow \infty} \sum_{j=1}^{N} T\left(\mathbb{1}_{E_{j}}\right) .
$$

The indicator function $\mathbb{1}_{F_{0}}$ is in $L^{p}$, and so by duality,

$$
\left\langle T\left(\mathbb{1}_{E}\right), \mathbb{1}_{F_{0}}\right\rangle_{q, p}=\lim _{N \rightarrow \infty}\left\langle\sum_{j=1}^{N} T\left(\mathbb{1}_{E_{j}}\right), \mathbb{1}_{F_{0}}\right\rangle_{q, p}=\lim _{N \rightarrow \infty} \sum_{j=1}^{N}\left\langle T\left(\mathbb{1}_{E_{j}}\right), \mathbb{1}_{F_{0}}\right\rangle_{q, p} .
$$

Therefore, $\mu\left(E, F_{0}\right)=\sum_{j=1}^{\infty} \mu\left(E_{j}, F_{0}\right)$, as required. Thus $\mu$ is a measure in each argument separately, and hence an $F_{2}$-measure.

Now we calculate the $F_{2}$-variation of $\mu$. Let $\left(E_{j}\right)$ and $\left(F_{k}\right)$ be finite collections of pairwise disjoint measurable sets, and for each $j$ and $k$ let $\epsilon_{j}$ and $\delta_{k}$ be arbitrary choices of signs. Then

$$
\begin{aligned}
\left|\sum_{j, k} \mu\left(E_{j}, F_{k}\right) \epsilon_{j} \delta_{k}\right| & =\left|\sum_{j, k}\left\langle T\left(\mathbb{1}_{E_{j}}\right), \mathbb{1}_{F_{k}}\right\rangle_{q, p} \epsilon_{j} \delta_{k}\right| \\
& =\left|\left\langle T\left(\sum_{j} \epsilon_{j} \mathbb{1}_{E_{j}}\right), \sum_{k} \delta_{k} \mathbb{1}_{F_{k}}\right\rangle_{q, p}\right| .
\end{aligned}
$$

By assumption, this is bounded by

$$
\left\|\sum_{j} \epsilon_{j} \mathbb{1}_{E_{j}}\right\|_{L^{p}(K)}\left\|\sum_{k} \delta_{k} \mathbb{1}_{F_{k}}\right\|_{L^{p}(K)} \leq 1 .
$$

This bound is uniform over choices of partitions and signs, and so $\|\mu\|_{F_{2}} \leq 1$, which provides the desired result.

Now it remains to verify 4.4. By Theorem 3.10, we have $\int\langle\boldsymbol{f}, \boldsymbol{g}\rangle_{H} d \mu=$ $\int\left\langle\boldsymbol{f}, d \nu_{\boldsymbol{g}}\right\rangle$, where

$$
\nu_{\boldsymbol{g}}(E)=\int \boldsymbol{g}(y) \mu(E, d y), \quad E \in \mathcal{F}
$$


By 4.5,

$$
\mu(E, d y)=\left(T \mathbb{1}_{E}\right) d \lambda,
$$

and so

$$
\nu_{\boldsymbol{g}}(E)=\int \boldsymbol{g}\left(T \mathbb{1}_{E}\right) d \lambda .
$$

By Lemma 3.8,

$$
\int\left\langle\boldsymbol{f}, d \nu_{\boldsymbol{g}}\right\rangle=\lim _{n \rightarrow \infty} \int\left\langle\boldsymbol{f}_{n}, d \nu_{\boldsymbol{g}}\right\rangle,
$$

where $\left(\boldsymbol{f}_{n}\right)$ is a sequence of uniformly bounded, countably valued measurable functions which converge uniformly to $\boldsymbol{f}$. (Such a sequence exists by Theorem 2.1.)

Suppose for a fixed $n \in \mathbb{N}, \boldsymbol{f}_{n}=\sum_{j=1}^{\infty} \boldsymbol{x}_{j} \mathbb{1}_{A_{j}}$. Then

$$
\int\left\langle\boldsymbol{f}_{n}, d \nu_{\boldsymbol{g}}\right\rangle=\sum_{j=1}^{\infty}\left\langle\boldsymbol{x}_{j}, \nu_{\boldsymbol{g}}\left(A_{j}\right)\right\rangle_{H} .
$$

By Lemma 3.3 and 4.6,

$$
\left\langle\boldsymbol{x}_{j}, \nu_{\boldsymbol{g}}\left(A_{j}\right)\right\rangle_{H}=\int\left\langle\boldsymbol{x}_{j}, \boldsymbol{g}\right\rangle_{H}\left(T \mathbb{1}_{A_{j}}\right) d \lambda .
$$

By the bilinearity of the inner product in $H$, and the linearity of $T$,

$$
\int\left\langle\boldsymbol{x}_{j}, \boldsymbol{g}\right\rangle_{H}\left(T \mathbb{1}_{A_{j}}\right) d \lambda=\int\left\langle T\left(\boldsymbol{x}_{j} \mathbb{1}_{A_{j}}\right), \boldsymbol{g}\right\rangle_{H} d \lambda .
$$

Therefore,

$$
\int\left\langle\boldsymbol{f}_{n}, d \nu_{\boldsymbol{g}}\right\rangle=\sum_{j=1}^{\infty} \int\left\langle T\left(\boldsymbol{x}_{j} \mathbb{1}_{A_{j}}\right), \boldsymbol{g}\right\rangle_{H} d \lambda .
$$

The Lebesgue dominated convergence theorem for Banach space-valued $L^{p}$-spaces (see [5, Theorem II.12.1]) implies that

$$
\sum_{j=1}^{N} \boldsymbol{x}_{j} \mathbb{1}_{A_{j}} \rightarrow \sum_{j=1}^{\infty} \boldsymbol{x}_{j} \mathbb{1}_{A_{j}}
$$

in $L^{p}(K, \lambda ; H)$ as $N \rightarrow \infty$. Therefore,

$$
\begin{aligned}
\sum_{j=1}^{\infty} \int\left\langle T\left(\boldsymbol{x}_{j} \mathbb{1}_{A_{j}}\right), \boldsymbol{g}\right\rangle_{H} d \lambda & =\int\left\langle T\left(\sum_{j=1}^{\infty} \boldsymbol{x}_{j} \mathbb{1}_{A_{j}}\right), \boldsymbol{g}\right\rangle_{H} d \lambda \\
& =\int\left\langle T\left(\boldsymbol{f}_{n}\right), \boldsymbol{g}\right\rangle_{H} d \lambda=\left\langle T\left(\boldsymbol{f}_{n}\right), \boldsymbol{g}\right\rangle_{q, p} .
\end{aligned}
$$

By the Lebesgue dominated convergence theorem for Banach space-val-

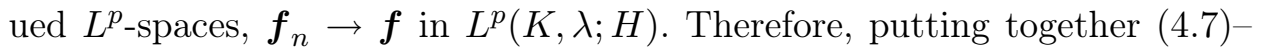
(4.9),

$$
\int\left\langle\boldsymbol{f}, d \nu_{\boldsymbol{g}}\right\rangle=\lim _{n \rightarrow \infty}\left\langle T\left(\boldsymbol{f}_{n}\right), \boldsymbol{g}\right\rangle_{q, p}=\langle T \boldsymbol{f}, \boldsymbol{g}\rangle_{q, p} .
$$

Therefore, $\int\langle\boldsymbol{f}, \boldsymbol{g}\rangle d \mu=\langle T \boldsymbol{f}, \boldsymbol{g}\rangle_{q, p}$, which completes the proof. 
Acknowledgements. The author wishes to thank R. C. Blei for suggesting this line of inquiry, and for his patience and guidance in the preparation of this paper.

\section{References}

[1] R. G. Bartle, A general bilinear vector integral, Studia Math. 15 (1956), 337-352.

[2] R. C. Blei, Analysis in Integer and Fractional Dimensions, Cambridge Stud. Adv. Math. 71, Cambridge Univ. Press, Cambridge, 2001.

[3] A. Bowers, A measure-theoretic Grothendieck inequality, J. Math. Anal. Appl. (2010), doi:10.1016/j.jmaa.2010.04.020.

[4] J. Diestel and J. J. Uhl, Jr., Vector Measures, Math. Surveys 15, Amer. Math. Soc., Providence, RI, 1977.

[5] N. Dinculeanu, Vector Measures, Int. Ser. Monogr. Pure Appl. Math. 95, Pergamon Press, Oxford, 1967.

[6] I. Dobrakov, On integration in Banach spaces. I, Czechoslovak Math. J. 20 (95) (1970), 511-536.

[7] N. Dunford, A bilinear vector integral. I, Adv. Math. 17 (1975), 337-342.

[8] A. Grothendieck, Sur les applications linéaires faiblement compactes d'espaces du type $C(K)$, Canad. J. Math. 5 (1953), 129-173.

[9] —, Résumé de la théorie métrique des produits tensoriels topologiques, Bol. Soc. Mat. São Paulo 8 (1956), 1-79.

[10] J. Lindenstrauss and A. Pełczyński, Absolutely summing operators in $\mathcal{L}_{p}$-spaces and their applications, Studia Math. 29 (1968), 275-326.

[11] J. Lindenstrauss and L. Tzafriri, Classical Banach Spaces II: Function Spaces, Springer, Berlin, 1979.

[12] B. J. Pettis, On integration in vector spaces, Trans. Amer. Math. Soc. 44 (1938), 277-304.

[13] W. Rudin, Real and Complex Analysis, McGraw-Hill, New York, 1987.

[14] K. Ylinen, On vector bimeasures, 3rd ed., Ann. Mat. Pura Appl. (4) 117 (1978), $115-138$.

Adam Bowers

Department of Mathematics

University of Missouri

Columbia, MO 65211, U.S.A.

E-mail: bowersad@missouri.edu 